This article was downloaded by:[Bernstein, Daniel M.]

On: 3 July 2007

[Bernstein, Daniel M.]

Access Details: [subscription number 780341012]

Publisher: Psychology Press

Informa Ltd Registered in England and Wales Registered Number: 1072954

Registered office: Mortimer House, 37-41 Mortimer Street, London W1T 3JH, UK

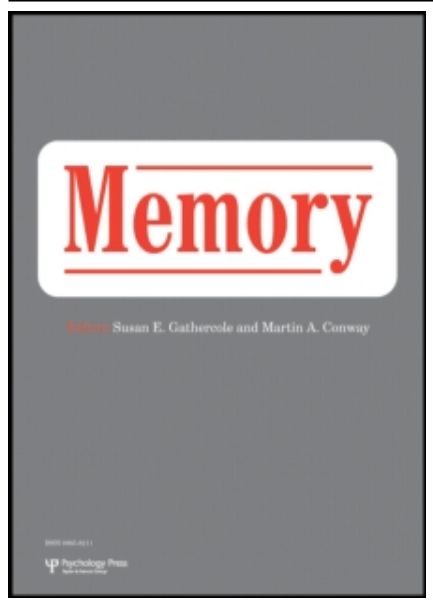

\title{
Memory
}

Publication details, including instructions for authors and subscription information: http://www.informaworld.com/smpp/title content=t713683358

\section{Fluency misattribution and visual hindsight bias}

Online Publication Date: 01 July 2007

To cite this Article: Bernstein, Daniel M. and Harley, Erin M. , (2007) 'Fluency

misattribution and visual hindsight bias', Memory, 15:5, 548 - 560

To link to this article: DOI: $10.1080 / 09658210701390701$

URL: http://dx.doi.org/10.1080/09658210701390701

PLEASE SCROLL DOWN FOR ARTICLE

Full terms and conditions of use: $\underline{\text { http://www.informaworld.com/terms-and-conditions-of-access.pdf }}$

This article maybe used for research, teaching and private study purposes. Any substantial or systematic reproduction, re-distribution, re-selling, loan or sub-licensing, systematic supply or distribution in any form to anyone is expressly forbidden.

The publisher does not give any warranty express or implied or make any representation that the contents will be complete or accurate or up to date. The accuracy of any instructions, formulae and drug doses should be independently verified with primary sources. The publisher shall not be liable for any loss, actions, claims, proceedings, demand or costs or damages whatsoever or howsoever caused arising directly or indirectly in connection with or arising out of the use of this material.

(C) Taylor and Francis 2007 


\title{
Fluency misattribution and visual hindsight bias
}

\author{
Daniel M. Bernstein \\ Kwantlen University College and University of Washington \\ Erin M. Harley \\ University of California, Los Angeles, and Exponent Failure Analysis Associates, Inc.
}

\begin{abstract}
We tested a fluency-misattribution theory of visual hindsight bias, and examined how perceptual and conceptual fluency contribute to the bias. In Experiment 1a observers identified celebrity faces that began blurred and then clarified (Forward baseline), or indicated when faces that began clear and then blurred were no longer recognisable (Backward baseline). In surprise memory tests that followed, observers adjusted the degree of blur of each face to match what the faces looked like when identified in the corresponding baseline condition. Hindsight bias was observed in the Forward condition: During the memory test observers adjusted the faces to be more blurry than when originally identified during baseline. These same observers did not show hindsight bias in the Backward condition: Here, they adjusted faces to the exact blur level at which they identified the faces during baseline. Experiment $1 \mathrm{~b}$ tested a combined condition in which faces were viewed in a Forward progression at baseline but in a Backward progression at test. Hindsight bias was observed in this condition but was significantly less than the bias observed in the Experiment 1a Forward condition. Experiments 1a and 1b provide support for the fluency-misattribution account of visual hindsight bias: When observers are made aware of why fluency has been enhanced (i.e., in the Backward condition) they are better able to discount it, and as a result show reduced or no hindsight bias. In Experiment 2, observers viewed faces in a Forward progression at baseline and then in a Forward upright or inverted progression at test. Hindsight bias occurred in both conditions, but was greater for upright than inverted faces. We conclude that both conceptual and perceptual fluency contribute to visual hindsight bias.
\end{abstract}

Early on the morning of 29 August 2005 hurricane Katrina made landfall in New Orleans, Louisiana, USA. Later that morning, the 17th Street Canal levee failed and water from Lake Pontchartrain surged into the already hurricane-beaten city. The next day a second levee was breached. In the weeks following the hurricane, debate ensued as to whether officials should have foreseen the failure of the levees: "I knew in my gut that this was the bad one," former FEMA director Michael Brown said, recalling his feelings from the day before Katrina hit (MSNBC NEWS, 2006). Prior to the storm, how accurately could the average person have estimated the likelihood of this outcome? Without the benefit of outcome knowledge, people make educated guesses about particular events; sometimes they are correct and sometimes they are not. In contrast, when making a judgement with the benefit of outcome knowledge, people make overly educated guesses. They tend to think that they "knew it all along" (Wood, 1978), and assume, for instance, that they would have predicted the breach of the levees in New Orleans. Such hindsight bias lends the world's events an air of inevitability.

Address correspondence to: Daniel M. Bernstein, University of Washington, Box 351525, Seattle, WA 98195, USA. E-mail: db6@u.washington.edu

We thank Aimee Ha for assistance in data collection. Geoff Loftus and Larry Sanna provided helpful comments on an earlier draft of this paper. This work was supported by NIMH Grant MH41637 to G. Loftus and a Kwantlen Faculty Professional Development grant to D. Bernstein. 
Hindsight bias occurs when outcome information biases one's judgement regarding the naive knowledge state of oneself or others (Fischhoff, 1975). The bias is robust and has been demonstrated for a variety of judgements. In a standard hindsight task conducted in the laboratory, participants predict the outcome to a problem or event prior to learning the outcome, e.g., "what will happen on President Nixon's 1972 visit to China and the Soviet Union." After learning the actual outcome, participants must try to recall their original predictions (or estimate the predictions given by others who do not have outcome information). Often, participants recall or estimate predictions that are closer to the actual outcome, thereby exhibiting hindsight bias (for a review, see Blank, Musch, \& Pohl, 2007).

\section{VISUAL HINDSIGHT BIAS AND FLUENCY MISATTRIBUTION}

Harley, Carlsen, and Loftus (2004) developed a visual analogue to the standard verbal hindsight bias task. Observers identified celebrity faces that began degraded (blurry) and then clarified on a computer screen (baseline condition). Following baseline, observers completed a hindsight condition in which they viewed the baseline faces again (old faces) plus a set of previously unseen faces (new faces), and tried to estimate the blur level at which a naive peer would be able to identify each face. In the hindsight condition faces clarified in the same manner as did those during baseline, except that each clarification sequence was preceded by a clear picture of that face (prime). Observers were asked to imagine that a same-age peer did not see the prime, and to stop the clarification when their peer would be able to identify the face. Hindsight bias was observed in that condition: Compared to baseline performance, observers overestimated the degradation level at which their peers would be able to identify the celebrity faces, i.e., they believed faces were identifiable at a blurrier point than was actually the case in the baseline task. Moreover, the magnitude of the bias was greater for old faces than for new faces. Harley et al. interpreted these results in terms of fluency misattribution, a brief explanation of which we now provide.

Fluency reflects the speed, ease, and accuracy with which a stimulus is processed. Although fluency can be enhanced via numerous stimulus variables-e.g., clarity, familiarity, and presenta- tion duration-participants are sometimes unaware of the cause or source of the enhancement. Under such conditions it has been shown that people can misattribute fluency. For example, Jacoby and Whitehouse (1989) found that when viewing words in a recognition test, participants unaware of subliminal prime words misattributed enhanced fluency of test words to prior exposure. Similarly, participants have been shown to misattribute enhanced fluency to a variety of stimulus judgements, e.g., truth value, liking, clarity, and duration (see Bernstein, Whittlesea, \& Loftus, 2002; Brinol, Petty, \& Tormala, 2006; Unkelbach, 2006; Winkielman, Schwarz, Reber, \& Fazendeiro, 2003). These studies provide evidence that people use the relative ease and speed of their information-processing experience to make cognitive, perceptual, and even affective judgements about the world.

Harley et al. (2004) proposed that fluency misattribution might account for visual hindsight bias. When observers know the identity of the blurry face a priori in the hindsight condition they process those faces more fluently. Unaware of why fluency has been enhanced they misattribute it to the predictability of the given outcome. Fluency misattribution predicts greater hindsight bias for old compared to new faces, and that is precisely what was found. While observers processed both face types fluently in hindsight as a result of viewing the primes prior to each clarification process, observers experienced additional fluency for old faces as a result of watching them clarify in the baseline condition. For both face types observers misattributed processing fluency to another person's knowledge, claiming that their naive peer was not, in fact, naive; however, they misattributed more for old than for new faces due to the enhanced fluency experienced for old faces.

As with any new finding, replication is warranted. Also, if fluency does in fact underlie the visual hindsight bias, as Harley et al. (2004) proposed, then it should be possible to eliminate or even reverse the bias under certain circumstances (see Roese, Fessel, Summerville, Kruger, \& Dilch, 2006).

\section{ELIMINATION AND REVERSAL OF HINDSIGHT BIAS}

Attempts to eliminate or reverse hindsight bias have been largely unsuccessful. For instance, 
Fischhoff (1977) found that warning participants about the dangers of hindsight failed to reduce bias. Similarly, Harley et al. (2004) educated participants about visual hindsight bias by explaining how participants usually perform a memory test when falling victim to the bias. The experimenters warned participants that they should try to avoid this bias when making their judgements and perform as accurately as possible. The education and warnings proved unsuccessful in reducing the bias. Thus, explicit warnings do not seem to be effective at reducing hindsight bias, suggesting the bias may be cognitively impenetrable.

More successful attempts to reduce or eliminate hindsight bias use implicit manipulations. For example, Sanna and colleagues (Sanna \& Schwarz, 2003; Sanna, Schwarz, \& Small, 2002) asked participants to think of either few or many thoughts about why a given outcome occurred (e.g., why the British won the British-Gurkha War of 1814). Participants asked to generate few thoughts found the task relatively easy. Conversely, participants asked to generate many thoughts found the task relatively difficult. Sanna and Schwarz refer to this relative ease or difficulty with which thoughts are brought to mind as "accessibility experience". Like fluency, accessibility experience describes the ease of one's processing experience, and can influence a variety of judgements (see, Winkielman et al., 2003). The concept of accessibility experience refers to the ease or difficulty of thought generation (or recall), whereas fluency refers to the ease or difficulty with which new information is processed. Thus, accessibility experience and fluency are different in terms of thought generation (or recall) versus processing, but they are similar in terms of inferences regarding quick versus slow experiences. As such, accessibility and fluency are both metacognitive experiences.

In Sanna et al.'s (2002) study, participants who had an easy time generating outcome-consistent thoughts rated the outcome as more foreseeable (i.e., exhibited greater hindsight bias) than those who had a difficult time. The latter participants interpreted their subjective experience of difficulty in generating many outcome-consistent thoughts as evidence that the given outcome was not foreseeable. Other work on attribution has demonstrated that providing participants with a viable source to explain processing fluency can dramatically affect cognitive and affective judgements (Oppenheimer, 2006; Sinclair, Mark, \& Clore, 1994).

Thus, it appears possible to eliminate or reverse hindsight bias. What is needed, though, is a subtle manipulation. In Experiments 1a and $1 b$, borrowing liberally from Jacoby and Whitehouse (1989), we sought to reduce, eliminate, or reverse the visual hindsight bias by making the source of enhanced fluency obvious to participants. In Experiment 2 we investigated whether conceptual fluency, perceptual fluency, or both contribute to visual hindsight bias.

\section{EXPERIMENT 1A}

In Experiment 1a observers identified celebrity faces in two conditions: Forward and Backward. Both conditions contained two parts: a baseline task and a memory test. The Forward condition was a replication of Harley et al.'s (2004) memory test design in which observers identified celebrity faces as they clarified over time (baseline) and then in a surprise memory test that followed, adjusted the blur level of each face to match how it looked when identified during baseline (see also Halberstadt \& Niedenthal, 2001). In the Backward condition baseline task, rather than start blurry and slowly clarify, each face began clear and slowly blurred. Observers identified the face at the outset and then indicated-while watching the face become blurrier over timewhen the celebrity was "no longer recognisable" (see Bernstein, Loftus, \& Meltzoff, 2005). For the Backward memory test, observers adjusted the blur level of each face to match how it looked when they claimed it was no longer recognisable during baseline. Mirroring their respective baseline conditions, the Forward memory test faces began blurry whereas the Backward memory test faces began clear.

Based on previous results (Bernstein, Atance, Loftus, \& Meltzoff, 2004; Harley et al., 2004), we expected that in the Forward condition observers would demonstrate hindsight bias by adjusting faces during the memory test to be more blurry than they were when identified during baseline. Based on Jacoby and Whitehouse's (1989) work, we expected that in the Backward condition observers would be aware of why fluency had been enhanced and therefore discount the fluency (and perhaps over-discount). Our rationale for why observers would be aware of the fluency and its source in the Backward condition was that the 
gradual blurring of the faces (from clear to less clear to blurry) would provide observers with an obvious source to which to attribute their fluency. The result of such discounting would be that in contrast to performance in the Forward condition, the Backward condition would result either in no hindsight bias (i.e., if observers stop the faces at the precise point that they had done previously), or a reverse hindsight bias (i.e., if observers stop the faces at a less degraded point than they had done previously). Like Jacoby and Whitehouse's Unaware and Aware groups, observers in our Forward and Backward conditions, respectively, would either not know or would know why they processed the faces fluently. When unaware of why fluency has been enhanced (Forward condition) observers would exhibit hindsight bias; conversely, when aware of why fluency had been enhanced (Backward condition) observers would exhibit no bias or a reverse hindsight bias.

The procedures used in Experiment 1a also provide for a test of the hysteresis effect. First reported by Bruner and Potter (1964), the hysteresis effect is when observers claim to perceive an image at a blurrier state when viewed in a clearto-blurry progression than when viewed in a blurry-to-clear progression. We expected that Experiment 1a observers would claim to perceive the identity of the celebrity faces at a blurrier state when viewed in the Backward condition than when viewed in the Forward condition.

\section{Method}

Observers. A total of 53 University of Washington undergraduates, all with normal or correctedto-normal vision, participated in exchange for course credit.

Apparatus. Data collection took place in a room equipped with four Macintosh eMac computers - each of which was equipped with a G4 processor and a 17-inch monitor-allowing up to four observers to participate in each data collection session. Curtains were hung between computers to prevent observers from viewing other monitors during the session. Each data collection session lasted approximately $30 \mathrm{~min}$ utes. The experiment was written and executed in MATLAB using the Psychophysics Toolbox extensions (Brainard, 1997; Pelli, 1997).

Stimuli. Stimuli were 36 greyscale pictures of celebrity faces. The set included well-known actors, musicians, politicians, and sports figures; for example, Jerry Seinfeld, Tom Hanks, Britney Spears, Hillary Clinton, and Michael Jordon. Each face measured 500 pixels from the bottom of the chin to the top of the head and subtended a visual angle of about $21.8^{\circ}$ vertically. The display monitor's background luminance was $2.94 \mathrm{~cd} / \mathrm{m}^{2}$.

For each face, 30 successively more blurred versions were created. Each blurred version was accomplished by Fourier transforming the image from pixel space into spatial-frequency space, multiplying the resulting frequency amplitude spectrum by a low-pass filter and inverse-Fourier transforming the result back into pixel space. The low-pass filter was designed such that for each blur level, it passed frequencies perfectly; in other words, it had a value of 1.0 up to some value of $f_{0}$ cycles per face height and then fell parabolically, reaching zero at the cut-off frequency value of $f_{1}=f_{0} \times 3$ cycles per face height. As $f_{0}$ and $f_{1}$ are made smaller, the filter cuts off more spatial frequencies, and the resulting face becomes blurrier, as demonstrated in Figure 1. In each of the three experiments reported here, $f_{1}$, the filter cut-off frequency, was used as the dependent variable - a measure of the degree of blur present in the image when the observer was able to (or believed he or she would be able to) identify the

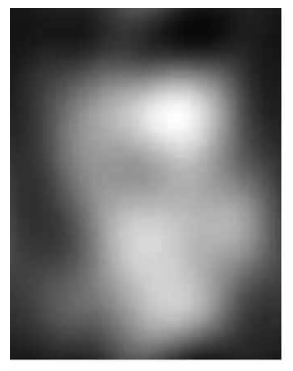

$\mathrm{f}_{1}=5.16$

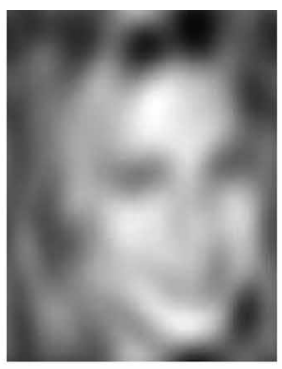

$\mathrm{f}_{1}=9.90$

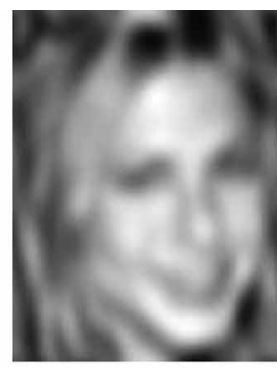

$\mathrm{f}_{1}=14.63$

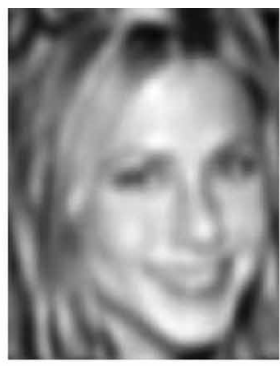

$\mathrm{f}_{1}=20.66$

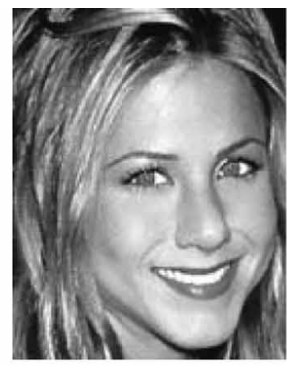

unfiltered

Figure 1. Sample of a celebrity face like those used in Experiments 1a-2. Shown is a subset of the 30 low-pass filtered images created for Jennifer Aniston with corresponding $\mathrm{f}_{1}$ (cycles per face height) filter cutoff values. 
celebrity. A blurrier picture is implied by a smaller $f_{1}$ value.

Design and procedure. Experiment 1a contained two conditions: Forward and Backward. Each condition had a baseline task and a memory test. Each observer completed both conditions. The Forward baseline task was a simple identification test. Half of the faces, 18 of 36, appeared in this condition. For each face, the 30 blurred images appeared, in order from most to least blurred, at a rate of $500 \mathrm{~ms}$ per image. To the observer, it looked as if the celebrity's face were slowly becoming clearer over time. For each face, observers were instructed to hit the space bar as soon as they recognised the celebrity, and then to type into the computer something that would identify the celebrity.

Observers were allowed to identify a celebrity in a number of ways, including any portion of the celebrity's name, the name of a character he or she plays on television, the name of a movie in which he or she has starred, and so on. Anything that indicated to the investigators that the observer recognised the celebrity was scored as an accurate response. At the completion of each trial, the observer verified his or her final identity guess. For each celebrity face, all 30 blurred images were displayed regardless of whether or when the face was identified. This was done to equate as best as possible the total time an observer viewed each face. The order in which the 18 celebrities appeared was randomised for each observer.

In the Backward baseline task faces began clear and slowly blurred; the 30 blurred images were displayed, in order from least to most blurred, at a rate of $500 \mathrm{~ms}$ per image. The 18 faces not shown in the Forward condition were shown in the Backward condition. Observers identified celebrities at the outset, and then indicated when the face was "no longer recognisable" by pressing the space bar to stop the blur sequence. Observers were allowed to stop the sequence multiple times if their decisions changed; this was done to prevent observers from stopping the sequence early to avoid missing a certain blur level. As in the Forward baseline task, all 30 blurred images were displayed regardless of whether or when the observer indicated that the face was no longer recognisable.

Following completion of the two baseline tasks observers received a surprise memory test (MT). Celebrities viewed in the Forward baseline condition were tested in the Forward MT (but in a different order), and celebrities viewed in the Backward baseline condition were tested in the Backward MT (also in a different order). For both memory tests, observers' task was to recall the degree of blur present when they stopped the blur sequence for the final time during the corresponding baseline task. For the Forward MT this was the point at which they first correctly identified the face, whereas for the Backward MT this was the point at which they last indicated that the face was no longer recognisable. The manner in which faces blurred or clarified in the memory tests was consistent with the manner in which they blurred or clarified during baseline presentation, so Forward MT faces began blurred and Backward MT faces began clear. In both tests each face was accompanied by the observer's identity response (regardless of whether or not it was correct), printed on the monitor just below the face. To adjust the degree of blur backwards or forwards, observers used the left and right arrow key respectively; a single key press changed the degree of blur by one step. They were allowed to range back and forth among the 30 filters until satisfied with their decisions. Once a satisfactory blur level was found observers hit the space bar to proceed to the next trial; no time limits were imposed.

Both baseline tasks were always completed prior to the memory tests. Condition order (i.e., whether Forward or Backward baseline was completed first), and choice of which celebrities were shown in Forward versus Backward conditions, were counterbalanced across observers. Two practice trials were completed prior to each of the four tasks; celebrities shown in practice trials did not appear in the experiment proper.

\section{Results}

Visual hindsight bias. Experiment 1a data are shown in Figure 2. In all experiments reported here, observers correctly identified at least $95 \%$ of the celebrities during baseline. Only trials for which an observer correctly identified the celebrity during baseline identification were included in the analysis. To determine whether observers exhibited hindsight bias in the Forward and Backward conditions we calculated a hindsight ratio (HR) for each condition. HR is the average blur level at which observers stopped faces during baseline divided by the average blur level to which observers adjusted faces during the 


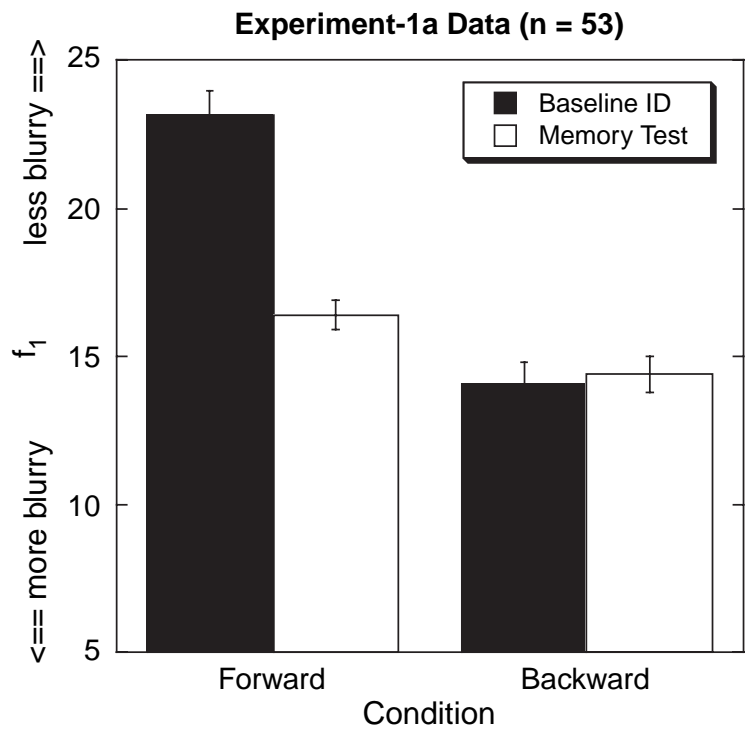

Figure 2. Experiment-1a data. Mean face-identification point (baseline or memory) is plotted as a function of $f_{1}$, filter cutoff frequency, expressed as cycles per face height. Error bars represent standard errors. Note: Forward-Condition faces were viewed in a blurry-to-clear progression whereas Backward-Condition faces were viewed in a clear-to-blurry progression.

memory test. We used $\mathrm{f}_{1}$, the filter cut-off frequency, as our measure of blur. A lower $\mathrm{f}_{1}$ value implies a blurrier face, so to the extent that observers show hindsight bias, HR will be greater than 1.0. Replicating Harley et al.'s (2004) result, we found that in the Forward condition observers, on average, adjusted faces in the memory test to be more blurry than the blur level at which they actually identified those faces during baseline $(\mathrm{HR}=1.46 \pm 0.10)$ (note that the notation $\mathrm{X} \pm \mathrm{Y}$ refers to a mean plus or minus a $95 \%$ confidence interval). In the Backward condition, though, we found no evidence of visual hindsight bias $(\mathrm{HR}=1.00 \pm 0.05)$ : During the memory test, observers, on average, stopped the celebrity faces at the same point at which they originally indicated that they could no longer recognise those faces during the baseline condition. Condition order had no effect on the data.

Hysteresis. Our data replicate the hysteresis effect (Bruner \& Potter, 1964), namely that observers claim to perceive an image at a blurrier state when viewed in a clear-to-blurry progression than in a blurry-to-clear progression. Consistent with this result, Experiment 1a observers stopped the celebrity faces at a blurrier point in the Backward baseline task than in the Forward baseline task, $t(52)=9.65, p<.001$. Thus, obser- vers indicated that they could identify blurring celebrity faces at a blurrier level than the level at which they could identify clarifying celebrity faces.

\section{Discussion}

The dissociation between performance in the Forward and Backward conditions lends further support to our claim that fluency misattribution underlies visual hindsight bias. To see how fluency misattribution can account for the present results, we discuss the Forward and Backward conditions in turn. In the Forward baseline task observers identified a series of celebrity faces as those faces began blurred and then clarified. Later, in the Forward memory test, observers indicated the point at which they stopped each face during baseline. Having already identified each of the faces previously, observers processed these faces fluently during the Forward memory test. However, unaware of the source of this fluency, observers misattributed the fluency to having identified the faces at a blurrier point than the point at which they truly identified those faces during baseline. The problem that observers encountered when making their hindsight judgements, we maintain, was that they were unaware of the reason why they could see and identify faces at a blurrier point during the memory test than during the baseline task.

The key to successful performance on the memory tests is to appreciate why one is processing the faces fluently. We argue that observers are unaware of the source of this fluency in the Forward condition, but aware of the source in the Backward condition. In the Backward baseline task, observers indicated when they could no longer recognise celebrity faces as those faces began clear and then blurred. Later, in the Backward memory test, observers indicated the point at which they stopped each face during baseline. As in the Forward condition just described, we believe that observers processed faces more fluently during the memory test than during the baseline task. However, unlike the Forward condition, observers in the Backward condition were fully aware of the source of their processing fluency. It is as if observers were being constantly reminded, on every frame, of why they were processing faces fluently during the Backward memory test. Observers knew the identity of the celebrity at the outset of each trial (as did 
observers at the outset of the Forward memory test trials), but they were also staring into the clear, un-degraded face of that celebrity. As the face blurred, observers processed the face fluently, but they were fully aware of the source of the fluency. Thus, observers knew precisely why they knew the identity of the celebrity face.

Hysteresis effects revealed different baseline performance levels for the Forward and Backward conditions; however, it is unlikely that differences in baseline can account for the differences in hindsight bias observed in the two conditions. Although the baseline identification point in the Backward condition was at a lower $\mathrm{f}_{1}$ value than was the identification point for the Forward condition (14.08 versus 23.12 respectively), the baseline $f_{1}$ in the Backward condition was not near floor levels. Of the 30 blur levels presented to observers, an $\mathrm{f}_{1}$ value of 14.08 is approximately step 14 . This means that observers could have exhibited hindsight bias had they chosen one of the 13 blur levels that was lower than step 14 during the Memory Test.

\section{EXPERIMENT 1B}

Traditional hindsight studies compare responses made in a naive knowledge state to responses made following the receipt of outcome information. In an effort to make the source of fluency obvious to observers in the Backward condition of Experiment 1a we departed from this traditional paradigm. Although observers did recall prior judgements, the prior judgements they were asked to recall were not made in a naive knowledge state. This is because the celebrities were shown clearly at the outset of the Backward baseline task. We therefore cannot rule out that any hindsight judgement would be unbiased when observers made their original judgements in a knowledgeable state. Experiment $1 \mathrm{~b}$ was designed to test whether, when recalling responses from a naive condition, hindsight bias could be reduced by making observers aware of fluency during the memory test.

To accomplish this, Experiment $1 \mathrm{~b}$ combined the Forward and Backward conditions of Experiment 1a: Observers viewed faces in a blurry-toclear (i.e., Forward) progression during baseline, but in a clear-to-blurry (i.e., Backward) progression during the memory test. The old-new effect in the Harley et al. (2004) study - greater bias for old than new faces-suggests that bias results from a combination of fluency that occurs during the baseline task, and fluency that occurs during the memory test (because observers showed more bias for faces shown in both conditions than faces shown only during the memory test). If bias results from enhanced fluency in both the baseline and memory test tasks, we reasoned that observers should discount the fluency that they experience when faces appear in a Backward progression during the memory test. Thus, we expected to reduce the amount of hindsight bias in Experiment $1 \mathrm{~b}$ in comparison to that found in the Forward condition of Experiment 1a (when bias resulted from viewing faces in both the baseline and memory test tasks).

\section{Method}

Observers. A total of 30 University of Washington undergraduates, all with normal or correctedto-normal vision, participated in exchange for course credit. None of the participants had participated in Experiment 1a.

Apparatus and stimuli. All equipment and stimuli were identical to those used in Experiment 1 a.

Design and procedure. All observers in Experiment $1 \mathrm{~b}$ viewed celebrities in a Forward baseline identification task followed by a Backward memory test. The baseline task was identical to the Forward baseline task used in Experiment 1a. Briefly, each face progressed from highly blurred to full clarity over 15 seconds, and observers stopped the resolution process as soon as they recognised the face. The memory test was similar to the Backward memory test used in Experiment 1a: Each face began clear and observers adjusted the level of blur until the face looked like it did when they stopped the resolution process and identified the face in the baseline task.

Celebrity order was counterbalanced across observers. Observers completed two practice trials prior to each of the two tasks. Celebrities shown in practice trials did not appear in the experiment proper.

\section{Results and discussion}

Experiment $1 \mathrm{~b}$ data are shown in Table 1. Data from Experiment 1a are reproduced in Table 1 for comparison. Only trials for which an observer correctly identified the celebrity during baseline 
TABLE 1

Comparison of hindsight effect sizes in Experiments $1 \mathrm{a}-\mathrm{b}$

\begin{tabular}{llll}
\hline & $\begin{array}{l}\text { Experiment 1a: } \\
\text { Backward condition }\end{array}$ & Experiment 1b & $\begin{array}{l}\text { Experiment 1a: } \\
\text { Forward condition }\end{array}$ \\
\hline $\mathrm{N}$ & 53 & 30 & 53 \\
Baseline condition & Backward & Forward & Forward \\
Test condition & Backward & Backward & Forward \\
Baseline ID $\mathrm{f}_{1}$ & 14.08 & 24.55 & 23.12 \\
Memory test $\mathrm{f}_{1}$ & 14.39 & 21.09 & 16.38 \\
Hindsight ratio $\pm 95 \% \mathrm{CI}$ & $1.00 \pm 0.05$ & $1.21 \pm 0.13$ & $1.46 \pm 0.10$ \\
\hline
\end{tabular}

Forward condition faces were shown in a blurry-to-clear progression, whereas Backward condition faces were shown in a clear-to-blurry progression. "Hindsight ratio" is the ratio of Baseline ID $\mathrm{f}_{1}$ to Memory test $\mathrm{f}_{1}$ averaged across participants. A ratio of 1.0 implies no hindsight bias; larger ratios imply larger hindsight bias effects.

identification were included in the analysis. The average hindsight ratio (HR) for Experiment $1 b$ - baseline $f_{1}$ divided by memory-test $f_{1}$ averaged across observers - revealed that observers did show hindsight bias $(\mathrm{HR}=1.21 \pm 0.13)$. To determine the relative size of the effect, Experiment $1 \mathrm{~b}$ data were compared to data from the Experiment 1a Forward and Backward conditions. The amount of hindsight bias exhibited in Experiment $1 \mathrm{~b}$ was less than that observed in the Forward condition of Experiment 1a, $t(81)=3.16, p<.01$, and more than that in the Backward condition of Experiment 1a, $t(81)=3.49, p<.001$.

As predicted by the fluency misattribution theory, hindsight bias was found in Experiment $1 \mathrm{~b}$, but the size of the bias was significantly less than that observed in the Forward condition of Experiment 1a. Experiment $1 \mathrm{~b}$ and the Forward condition in Experiment 1a used identical forward baseline tasks, i.e., observers identified blurry faces as they clarified over time. In both experiments observers were then given a surprise memory test and adjusted the degree of blur on each face until the face looked like it did when identified during baseline. In both experiments observers were allowed to toggle back and forth among the 30 blur levels until satisfied with their decisions. The only difference between the two protocols was that in Experiment 1a the memory test faces began blurry, and in Experiment $1 \mathrm{~b}$ they began clear. Despite this relatively small difference in protocol, a significant difference was found between hindsight effect sizes in the two experiments, with a larger bias occurring when the memory test faces began blurry.

\section{EXPERIMENT 2}

In Experiments 1a and $1 \mathrm{~b}$ there were two sources of fluency that observers had to discount if they were to accurately recall baseline performance: perceptual and conceptual fluency. Perceptual fluency in the present study arises when observers see faces clarify or blur in the same manner in which they saw those faces clarify or blur previously. Conceptual fluency arises when observers know the identity of the faces prior to seeing the face clarify or blur. In one prior study, Werth and Strack (2003) found that perceptual fluency increases hindsight bias. In their paradigm, participants read trivia questions that were either easy to see (high perceptual fluency) or hard to see (low perceptual fluency). As expected, the easier it was to read the question, the more hindsight bias participants showed. This finding demonstrates the role of perceptual fluency in hindsight bias; however, these authors did not assess the role of conceptual fluency in their study.

In Experiments $1 \mathrm{a}-\mathrm{b}$ in the current work, and in Harley et al.'s (2004) study, conceptual and perceptual fluency were both present. Thus, we do not yet know which type of fluency is producing visual hindsight bias. In Experiment 2 we explored whether visual hindsight bias results from perceptual fluency, conceptual fluency, or a combination of the two.

We manipulated perceptual fluency in Experiment 2 by showing faces either upright or inverted at test. Inverting a face is detrimental to face recognition (see e.g., Loftus, Oberg, \& Dillon, 2004; Valentine \& Bruce, 1986). Observers typically process upright faces holistically, whereby facial features (e.g., eyes, mouth, nose) 
are processed fluently and perceived as a wellintegrated whole. Conversely, observers process inverted faces feature by feature, whereby facial features are not processed fluently and not perceived as a well-integrated whole (see e.g., Diamond \& Carey, 1986; Farah, Wilson, Drain, \& Tanaka, 1998; Hole, 1994; Rhodes, Brake, \& Atkinson, 1993). Therefore, one way to dramatically impair perceptual fluency of a face is to present the face inverted.

In Experiment 2 observers completed a baseline identification task followed by a memory test identical to the protocol of the Forward condition of Experiment 1a, but with one difference: During the memory test, half of the faces were presented upright (as in baseline) and half were presented inverted. For both upright and inverted faces the celebrity's name was printed below the face to give conceptual priming. We know from the results of Experiment 1a and previous studies (Harley et al., 2004) that hindsight bias will be found for upright faces. Whether a bias occurs for inverted faces, and if so, its magnitude compared to the bias observed for upright faces, will reveal the relative contributions of perceptual and conceptual fluency to visual hindsight bias.

If perceptual fluency alone underlies the bias, then we expect upright faces to produce ample hindsight bias, but inverted faces to produce little or no hindsight bias. The reason is that for both upright and inverted faces, conceptual fluency is constant (present in the name prime); however, perceptual fluency varies as previously discussed. Observers should experience much perceptual fluency for upright faces, but little to no perceptual fluency for inverted faces. If inverted faces produce no visual hindsight bias, then we would conclude that perceptual fluency alone underlies visual hindsight bias. In contrast, if conceptual fluency alone underlies visual hindsight bias, then we expect upright and inverted faces to produce equal amounts of hindsight bias. Again, the reason is that conceptual fluency remains constant for upright and inverted faces, but perceptual fluency varies. Note, however, that if equal bias were observed for upright and inverted faces we could not rule out the possibility that perceptual fluency is contributing to the bias for upright faces, but that it interacts with conceptual fluency. Such an interaction could theoretically cause the individual effects to be reduced so that the total bias would not exceed the effect of conceptual fluency acting alone on the inverted faces. Finally, if perceptual and conceptual fluency both contribute to visual hindsight bias, then we expect both upright and inverted faces to produce hindsight bias - the reason is that conceptual fluency accompanies both orientations-but we expect to see greater bias for upright than inverted faces, because upright faces also have perceptual fluency.

\section{Method}

Observers. A total of 42 University of Washington undergraduates, all with normal or correctedto-normal vision, participated in exchange for course credit. None of the participants had participated in Experiment 1a or $1 \mathrm{~b}$.

Apparatus and stimuli. All equipment and stimuli were identical to those used in Experiments $1 \mathrm{a}-\mathrm{b}$.

Design and procedure. Experiment 2 consisted of two tasks: baseline identification and memory test. The baseline identification task was identical to the Forward baseline condition in Experiment 1a. Briefly, each face progressed from highly blurred to full clarity over 15 seconds, and observers stopped the resolution process as soon as they recognised the face.

During the memory test, faces began blurry and the observer's response from baseline was printed on the screen. Observers used the arrow keys to adjust the degree of blur until the face looked like it did when identified during baseline. As in Experiments $1 \mathrm{a}-\mathrm{b}$, observers were allowed to move back and forth among the 30 filters; no time limits were imposed. Critically, unlike Experiments $1 \mathrm{a}-\mathrm{b}$, during the Experiment 2 memory test half of the faces appeared upright and half appeared inverted. Presentation of upright and inverted faces during test was mixed across trials.

Celebrity order and the choice of which celebrities appeared upright and which appeared inverted at test were counterbalanced across observers. Observers completed two practice trials prior to each of the two tasks. Celebrities shown in practice trials did not appear in the experiment proper.

\section{Results and discussion}

Only trials for which an observer correctly identified the celebrity during baseline identification were included in the analysis. Observers 
exhibited hindsight bias for both upright faces $(\mathrm{HR}=1.39 \pm 0.09)$ and inverted faces $(\mathrm{HR}=$ $1.23 \pm 0.09)$. Our primary question was whether inverted faces would produce less, equal, or more bias than upright faces. As can be seen in Figure 3, upright faces produced more bias than inverted faces, $t(41)=3.68, p<.001$. We therefore conclude that perceptual and conceptual fluency both contribute to visual hindsight bias.

\section{GENERAL DISCUSSION}

These experiments demonstrate the role of fluency in visual hindsight bias. In Experiment 1a, observers identified celebrity faces as those faces began blurred and then clarified (Forward baseline), or they indicated when they could no longer identify celebrity faces as the faces began clear and then blurred (Backward baseline). Next, observers completed a memory test in which they viewed the faces blurring or clarifying in the same manner in which they had during baseline. Observers tried to recall the precise point at which they stopped each face during baseline. As we expected, when the faces began blurred and clarified for the second time, observers overestimated their naive knowledge by stopping the faces at a blurrier point than they

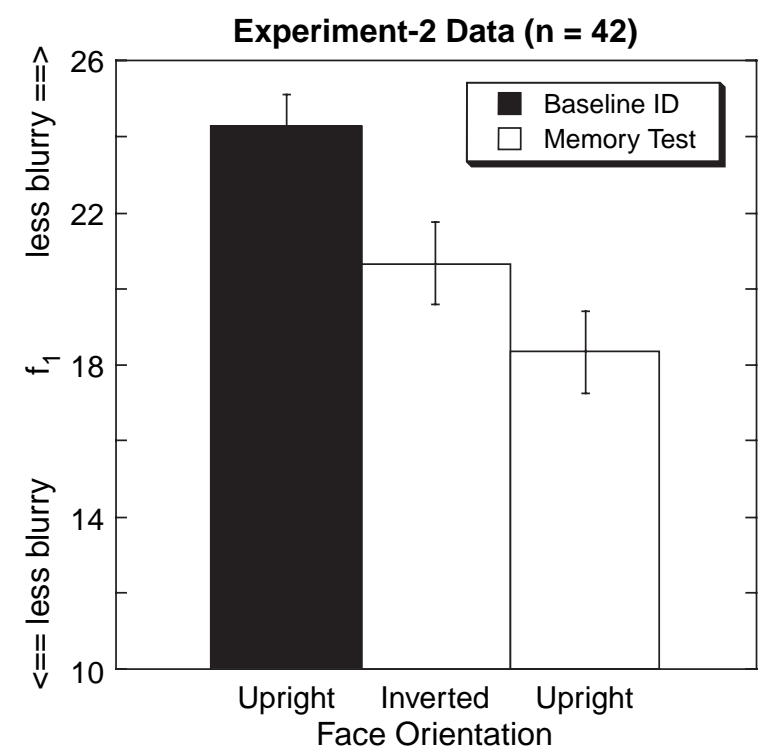

Figure 3. Experiment-2 data. Mean face-identification point (baseline or memory) is plotted as a function of $f_{1}$, filter cutoff frequency, expressed as cycles per face height. Error bars represent standard errors. had done previously (visual hindsight bias). However, observers did not overestimate their original judgements when the faces began clear and then blurred for the second time (no visual hindsight bias). Here, observers correctly recalled the point at which they had initially stopped the faces. Experiment $1 \mathrm{~b}$ tested a combined condition in which observers completed the naive Forward baseline task followed by the Backward memory test. Here, observers demonstrated an intermediate amount of bias compared to the Forward and Backward conditions from Experiment 1a. In Experiment 2, observers completed the Forward baseline condition and then completed a Forward memory test in which faces appeared upright or inverted. Observers showed visual hindsight bias for both types of faces; however, they showed more bias for upright than for inverted faces.

We designed these experiments to test the fluency misattribution theory of visual hindsight bias (Harley et al., 2004). Visual hindsight bias, like ordinary verbal hindsight bias, occurs when observers know the outcome to a problem, and then overestimate their own or another person's naive knowledge of the problem. For example, observers who know the identity of a degraded picture (e.g., of a celebrity face or a common object) will overestimate their own or another's naive knowledge of the picture (Bernstein et al., 2004; Harley et al., 2004). Thus, privileged knowledge biases people's judgement of naive knowledge (Hawkins \& Hastie, 1990; Wilson \& Brekke, 1994). According to the fluency misattribution theory of visual hindsight bias, observers process the identity of a picture more fluently when they know the identity of the picture. Like priming, prior knowledge of a picture's identity speeds one's processing of the picture. This enhanced speed of processing (fluency) can be perceptual, conceptual or both (see Whittlesea, 1993). Thus, knowing that a degraded picture represents the politician Bill Clinton leads people to process the picture more fluently, which, in turn, they mistakenly attribute to their own or another's naive knowledge.

Fluency misattribution can account for the present results as follows: When observers know in advance that they will be viewing a particular celebrity face, they process degraded forms of that face fluently. In cases where the face begins blurred and then clarifies, observers misattribute their processing fluency to having identified the face at a more degraded state than they had 
actually done previously. We call this visual hindsight bias (Harley et al., 2004). In cases where the face begins clear and then blurs during both baseline and test, observers correctly discount their processing fluency and avoid hindsight bias. What we find most striking about this dissociation is that the magnitude of the visual hindsight bias is very large in the former case and non-existent in the latter case. It is quite difficult to find evidence in the memory literature of nearperfect memory for a large set of stimuli, yet our observers demonstrated excellent memory in our backward-identification condition.

What is unclear from these results, though, is whether observers actually remembered the precise point at which they had initially stopped the image of a blurring celebrity face. Two additional possibilities for why we observed near-perfect memory in the Backward condition of Experiment 1a are as follows: (1) observers simply performed the same task twice, and thus were able to stop the face when they no longer could identify it in both the baseline and memory condition; (2) like the visual hindsight bias that observers displayed in the Forward condition, their perfect memory in the Backward condition was also the product of bias. According to the latter possibility, by discounting fluency, observers in the Backward memory test condition could arrive at the correct level of blur for a particular face without actually remembering that they had stopped the face at this point previously. In signal detection theory terms, observers adopted a liberal bias in the Forward memory test condition and a conservative bias in the Backward memory test condition.

In Experiment 2 we investigated the relative contributions of perceptual and conceptual fluency to visual hindsight bias. We reasoned that inverting memory test faces would eliminate or drastically reduce perceptual fluency, while keeping conceptual fluency constant. We again found hindsight bias for upright faces, replicating Experiment 1a and prior work (Harley et al., 2004). We also found bias for inverted faces; however, the magnitude of this bias was smaller than that for upright faces. These results lead us to two conclusions. First, perceptual and conceptual fluency both contribute to visual hindsight bias. Second, perceptual fluency enhances the bias above and beyond conceptual fluency alone.

\section{Alternative theories}

We argue that traditional theories of hindsight bias cannot account for the differences in bias effect sizes observed in the studies reported here. For example, cognitive reconstruction theories argue that bias occurs as a result of original evidence being reanalysed and reworked following the receipt of outcome information (for a review see, Hawkins \& Hastie, 1990). Through this rejudgement process, evidence consistent with the outcome is elaborated whereas evidence inconsistent with the outcome is minimised or discounted. Experiments 1a, 1b, and 2 contained the identical naive foresight task (Forward condition). Because observers watched each face clarify fully in the baseline task, outcome information was also constant across the experiments. Memory reconstruction should occur following the receipt of outcome information and should not be influenced by the mode of presentation during the memory test; therefore, cognitive reconstruction theories should predict equal bias for Forward and Backward memory tests, and equal bias for upright and inverted faces at test. In contrast, mode of presentation has a large effect on how fluently the faces are processed during the memory test; therefore fluency misattribution predicts larger bias in the Forward memory test design compared to the Backward design, and larger bias for upright than inverted faces, consistent with the data reported here.

Another popular theory of hindsight bias, anchoring and adjustment, posits that observers anchor on the outcome to a problem or question and then adjust their hindsight estimate to accommodate this anchor. Similar to the argument above, adjustment should be made immediately following receipt of outcome information, and should not be influenced by the mode of presentation at test. So, like cognitive reconstruction theories, anchoring and adjustment would predict equal bias for the Forward and Backward memory tests, as well as for upright and inverted faces.

According to another more general memory theory, transfer-appropriate processing (TAP), memory improves to the extent that information processing present at retrieval matches that at encoding (Masson \& MacLeod, 1992; Morris, Bransford, \& Franks, 1977; Rajaram, Srinivas, \& Roediger, 1998). First, TAP would predict better 
memory performance, i.e., less hindsight bias, for the Forward condition from Experiment 1awhere both baseline and memory test faces were shown in the Forward condition - than for the Experiment 1b design in which baseline faces were presented in a Forward condition but memory test faces were presented in a Backward condition. The opposite result was obtained. Second, TAP would predict better memory performance for upright than inverted faces in Experiment 2, because upright faces appear in the same orientation in which they appeared during baseline. Again, the opposite result was obtained.

\section{Future directions}

A question that remains unanswered is whether visual hindsight bias and the more traditional verbal hindsight bias are components of the same general bias-i.e., are we studying one phenomenon or many? Previous work using verbal materials provides what we think is a limited view of hindsight's generality. Preliminary evidence that hindsight bias is a general phenomenon that spans multiple sensory modalities comes from work in which preschoolers completed a real-world version of the visual hindsight bias task (with real objects hidden behind filter screens) in addition to verbal hindsight bias questions (Bernstein, Atance, Meltzoff, \& Loftus, in press). In that work, visual hindsight bias correlated with verbal hindsight bias, indicating that the two are related. Future work should investigate to what extent visual and verbal hindsight bias correlate in adults, and in particular the role of fluency misattribution in verbal hindsight bias. Further, if visual and verbal hindsight bias stem from the same general phenomenon, we should also find evidence of hindsight bias in other sensory modalities including olfaction and audition (see Pohl, 2007).

Manuscript received 26 June 2006 Manuscript accepted 25 March 2007 First published online 31 May 2007

\section{REFERENCES}

Bernstein, D. M., Atance, C., Loftus, G. R., \& Meltzoff, A. N. (2004). We saw it all along: Visual hindsight bias in children and adults. Psychological Science, $15,264-267$.
Bernstein, D. M., Atance, C., Meltzoff, A. N., \& Loftus, G. R. (in press). Hindsight bias and developing theories of mind. Child Development.

Bernstein, D. M., Loftus, G. R., \& Meltzoff, A. N. (2005). Object identification in preschool children and adults. Developmental Science, 8, 151-161.

Bernstein, D. M., Whittlesea, B. W. A., \& Loftus, E. F. (2002). Increasing confidence in remote autobiographical memory and general knowledge: Extensions of the revelation effect. Memory \& Cognition, 30, 432-438.

Blank, H., Musch, J., \& Pohl, R. F. (2007). Hindsight bias: On being wise after the event. Social Cognition, 25, 1-9.

Brainard, D. H. (1997). The psychophysics toolbox. Spatial Vision, 10, 433-436.

Brinol, P., Petty, R. E., \& Tormala, Z. L. (2006). The malleable meaning of subjective ease. Psychological Science, 17, 200-206.

Bruner, J. S., \& Potter, M. C. (1964). Interference in visual recognition. Science, 144, 424-425.

Diamond, R., \& Carey, S. (1986). Why faces are and are not special: An effect of expertise. Journal of Experimental Psychology: General, 115, 107-117.

Farah, M. J., Wilson, K. D., Drain, M., \& Tanaka, J. N. (1998). What is "special" about face perception? Psychological Review, 105, 482-498.

Fischhoff, B. (1975). Hindsight does not equal foresight: The effect of outcome knowledge on judgment under uncertainty. Journal of Experimental Psychology: Human Perception and Performance, 1, 288-299.

Fischhoff, B. (1977). Perceived informativeness of facts. Journal of Experimental Psychology: Human Perception and Performance, 3, 349-358.

Halberstadt, J. B., \& Niedenthal, P. M. (2001). Effects of emotion concepts on perceptual memory of emotional expressions. Journal of Personality and Social Psychology, 81, 587-598.

Harley, E. M., Carlsen, K. A., \& Loftus, G. R. (2004). The "saw-it-all-along" effect: Demonstrations of visual hindsight bias. Journal of Experimental Psychology: Learning, Memory, and Cognition, 30, 960-968.

Hawkins, S., \& Hastie, R. (1990). Hindsight: Biased judgments of past events after the outcomes are known. Psychological Bulletin, 107, 311-327.

Hole, G. J. (1994). Configurational factors in the perception of unfamiliar faces. Perception, 23, 65-74.

Jacoby, L. L., \& Whitehouse, K. (1989). An illusion of memory: False recognition influenced by unconscious perception. Journal of Experimental Psychology: General, 118, 126-135.

Loftus, G. R., Oberg, M. A., \& Dillon, A. M. (2004). Linear theory, dimensional theory, and the faceinversion effect. Psychological Review, 111, 835-865.

Masson, M. E., \& MacLeod, C. M. (1992). Reenacting the route to interpretation: Enhanced perceptual identification without prior perception. Journal of Experimental Psychology: General, 121, 145-176.

Morris, C. D., Bransford, J. D., \& Franks, J. J. (1977). Levels of processing versus transfer appropriate 
processing. Journal of Verbal Learning \& Verbal Behavior, 16, 519-533.

MSNBC News. (2006). Katrina the long road back. Retrieved 6 June 2006 from: http://www.msnbc.msn. com/id/11255379/

Oppenheimer, D. M. (2006). Consequences of erudite vernacular utilized irrespective of necessity. Applied Cognitive Psychology, 20, 139-156.

Pelli, D. G. (1997). The VideoToolbox software for visual psychophysics: Transforming numbers into movies. Spatial Vision, 10, 437-442.

Pohl, R. F. (2007). Ways to assess hindsight bias. Social Cognition, 25, 14-31.

Rajaram, S., Srinivas, K., \& Roediger, H. L. (1998). A transfer-appropriate processing account of context effects in word-fragment completion. Journal of Experimental Psychology: Learning, Memory, and Cognition, 24, 993-1004.

Rhodes, G., Brake, S., \& Atkinson, A. P. (1993). What's lost in inverted faces? Cognition, 47, 25-57.

Roese, N. J., Fessel, F., Summerville, A., Kruger, J., \& Dilich, M. A. (2006). The propensity effect: When foresight trumps hindsight. Psychological Science, $17,305-310$.

Sanna, L. J., \& Schwarz, N. (2003). Debiasing the hindsight bias: The role of accessibility experiences and (mis)attributions. Journal of Experimental Social Psychology, 39, 287-295.

Sanna, L. J., Schwarz, N., \& Small, E. M. (2002). Accessibility experiences and the hindsight bias: I knew it all along versus it could never have happened. Memory \& Cognition, 30, 1288-1296.

Sinclair, R. C., Mark, M. M., \& Clore, G. L. (1994). Mood-related persuasion depends on (mis)attributions. Social Cognition, 12, 309-326.

Unkelbach, C. (2006). The learned interpretation of cognitive fluency. Psychological Science, 17, $339-345$

Valentine, T., \& Bruce, V. (1986). The effect of race, inversion and encoding activity upon face recognition. Acta Psychologica, 61, 259-273.

Werth, L., \& Strack, F. (2003). An inferential approach to the knew-it-all-along phenomenon. Memory, 11, 411-419.

Whittlesea, B. W. A. (1993). Illusions of familiarity. Journal of Experimental Psychology: Learning, Memory, and Cognition, 19, 1235-1253.

Wilson, T. D., \& Brekke, N. (1994). Mental contamination and mental correction: Unwanted influences on judgements and evaluations. Psychological Bulletin, 116, 117-142.

Winkielman, P., Schwarz, N., Reber, R., \& Fazendeiro, T. A. (2003). Cognitive and affective consequences of visual fluency: When seeing is easy on the mind. In L. M. Scott \& R. Batra (Eds.), Persuasive imagery: A consumer response perspective (pp. 75-89). Mahwah, NJ: Lawrence Erlbaum Associates Inc.

Wood, G. (1978). The knew-it-all-along effect. Journal of Experimental Psychology: Human Perception and Performance, 4, 345-353. 